\title{
Electrical conductivity of hot and dense QCD matter created in heavy-ion collisions: A color string percolation approach
}

\author{
Pragati Sahoo, Swatantra Kumar Tiwari, and Raghunath Sahoo ${ }^{\dagger}$ \\ Discipline of Physics, School of Basic Sciences, Indian Institute of Technology Indore, \\ Indore 453552, India
}

(Received 28 April 2018; revised manuscript received 12 July 2018; published 6 September 2018)

\begin{abstract}
Recently, transport coefficients, viz., shear viscosity, electrical conductivity, etc., of strongly interacting matter produced in heavy-ion collisions have drawn considerable interest. We study the normalized electrical conductivity $\left(\sigma_{\mathrm{el}} / \mathrm{T}\right)$ of hot QCD matter as a function of temperature $(\mathrm{T})$ using the color string percolation model (CSPM). We also study the temperature dependence of shear viscosity and its ratio with electrical conductivity for the QCD matter. We compare CSPM estimations with various existing results and lattice QCD predictions with $(2+1)$ dynamical flavors. We find that $\sigma_{\mathrm{el}} / \mathrm{T}$ in CSPM has a very weak dependence on the temperature. We compare CSPM results with those obtained in the Boltzmann approach to multiparton scatterings model. A good agreement is found between CSPM results and predictions of the Boltzmann approach to multiparton scatterings with a fixed strong coupling constant.
\end{abstract}

DOI: 10.1103/PhysRevD.98.054005

\section{INTRODUCTION}

Ultrarelativistic heavy-ion collision programs at the Relativistic Heavy-Ion Collider (RHIC) and Large Hadron Collider (LHC) produce a strongly interacting matter known as quark-gluon plasma (QGP) [1]. Various experimental studies have been done in order to characterize the properties and behavior of matter at extreme conditions of temperature and energy densities. The transport properties are very important in understanding the evolution of the strongly interacting matter produced in heavy-ion collisions. The transport properties are mainly the theoretical inputs to the hydrodynamical calculations and affect various observables such as elliptic flow, transverse momentum spectra of particles created in heavy-ion collisions [2-4]. A very small shear viscosity-to-entropy density ratio explains the elliptic flow of identified hadrons produced at RHIC and LHC energies [5] and suggests the fluidity of the hot QCD matter produced. Various methods are used to estimate the shear viscosity $(\eta)$ such as Kubo formalism [6], effective models [7-13], etc.

Electrical conductivity $\left(\sigma_{\mathrm{el}}\right)$ is another key transport coefficient in order to understand the behavior and properties

\footnotetext{
*sktiwari4bhu@gmail.com

${ }^{\dagger}$ Corresponding author.

raghunath.sahoo@cern.ch

Published by the American Physical Society under the terms of the Creative Commons Attribution 4.0 International license. Further distribution of this work must maintain attribution to the author(s) and the published article's title, journal citation, and DOI. Funded by SCOAP .
}

of strongly interacting matter. This plays an important role in the hydrodynamic evolution of the matter produced in heavy-ion collisions in which charge relaxation takes place. In Ref. [14], the electrical conductivity is extracted from charge-dependent flow parameters from asymmetric heavyion collisions. Experimentally, it has been observed that very strong electric and magnetic fields are created in the early stages $(1-2 \mathrm{fm} / \mathrm{c})$ of noncentral collisions of nuclei at the RHIC and LHC $[14,15]$. The values of the electric and magnetic fields at the RHIC are $\mathrm{eE} \approx m_{\pi}^{2} \approx 10^{21} \mathrm{~V} / \mathrm{cm}$ and $\mathrm{eB} \approx m_{\pi}^{2} \approx 10^{18} \mathrm{G}$ [15]. Such a large electrical field influences the medium, which depends on the electrical conductivity. $\sigma_{\mathrm{el}}$ is responsible for producing an electric current in the early stage of the heavy-ion collision.

With the prior knowledge of color charges and the associated electric charges of the quarks, one might presume the QCD matter to be highly conductive. In contrast, this assumption fails due to the high interaction rates of the produced QCD matter, which again suggests a low shear viscosity-to-entropy density ratio $(\eta / s)$. In highly conducting quark-gluon plasma, the screening of external electromagnetic fields happens due to the high values of $\sigma_{\mathrm{el}}$ like the Meissner effect in superconductors as well as the "skin effect" for the electric current [16]. The electrical conductivity is one of the fundamental reasons for chiral magnetic effect [17], which is a signature of $C P$ violation in the strong interaction. In view of this, a detailed study of electrical conductivity in the strongly interacting QCD matter is inevitable.

The experimental measurement of electrical conductivity $\left(\sigma_{\mathrm{el}}\right)$ of the matter produced in heavy-ion collisions is not possible. Its information can be extracted from flow 
parameters measured in heavy-ion collision experiments [14]. Recently, various theoretical approaches have been used to study the electrical conductivity $[16,18-31] . \sigma_{\mathrm{el}}$ is also related to the soft dilepton production rate [32] and the magnetic field diffusion in the medium $[33,34]$.

The color string percolation model (CSPM) is a QCDinspired model [35-39], which can be used as an alternative approach to a color glass condensate. In the CSPM, the color flux tubes are stretched between the colliding partons in terms of the color field. The strings produce a $q \bar{q}$ pair in finite space filled similarly as in the Schwinger mechanism of pair creation in a constant electric field covering all the space [40]. With the growing energy and the number of nucleons of participating nuclei, the number of strings grows. Color strings may be viewed as small disks in the transverse space filled with the color field created by colliding partons. The number of strings grows as the energy and size of the colliding nuclei increase and starts overlapping to form clusters. After a critical string density is reached, a macroscopic cluster that marks the percolation phase transition, which spans the transverse nuclear interaction area, appears. Two-dimensional (2D) percolation is a nonthermal second-order phase transition. In the CSPM, the Schwinger barrier penetration mechanism for particle production, the fluctuations in the associated string tension, and the quantum fluctuations of the color fields make it possible to define a temperature. Consequently, the particle spectrum is produced with a thermal distribution. When the initial density of interacting colored strings $(\xi)$ exceeds the 2D percolation threshold $\left(\xi_{c}\right)$, i.e., $\xi>\xi_{c}$, a macroscopic cluster, which defines the onset of color deconfinement, appears. The critical density of percolation is related to the effective critical temperature, and thus percolation may be a possible way to achieve deconfinement in ultrarelativistic heavy-ion collisions [41] and in high-multiplicity pp collisions $[42,43]$. It is observed that the CSPM can be successfully used to describe the initial stages in highenergy heavy-ion collisions [40]. Recently, we have used CSPM to perform the energy and centrality dependent study of the deconfinement phase transition at RHIC Beam Energy Scan (BES) energies [44]. We have also studied various thermodynamical and transport properties at RHIC BES energies in this approach [45].

In this work, for the first time, we give the formulation of $\sigma_{\mathrm{el}}$ in the color string percolation approach. The paper is organized as follows. In Sec. II, we give the detailed formulation for calculation of electrical conductivity and shear viscosity in the CSPM, and we present results and discussions in Sec. III. Finally, we present a summary and conclusions in Sec. IV.

\section{ELECTRICAL CONDUCTIVITY AND SHEAR VISCOSITY}

In this section, we develop the formulation for evaluating the electrical conductivity of strongly interacting matter using the color string percolation approach. We start with few basic equations of the CSPM. The percolation density parameter, $\xi$, for central $\mathrm{Au}+\mathrm{Au}$ collisions at RHIC energies is calculated by using the parametrization of $\mathrm{pp}$ collisions at $\sqrt{s}=200 \mathrm{GeV}$ as discussed below. In the CSPM, one obtains

$$
\frac{d N_{\mathrm{ch}}}{d p_{\mathrm{T}}^{2}}=\frac{a}{\left(p_{0}+p_{\mathrm{T}}\right)^{\alpha}},
$$

where a is the normalization factor and $p_{0}$ and $\alpha$ are fitting parameters given as $p_{0}=1.982$ and $\alpha=12.877$ [46]. Because of the low string overlap probability in $\mathrm{pp}$ collisions, the fit parameters are then used to evaluate the interactions of the strings in $\mathrm{Au}+\mathrm{Au}$ collisions as

$$
p_{0} \rightarrow p_{0}\left(\frac{\left\langle n S_{1} / S_{n}\right\rangle_{\mathrm{Au}+\mathrm{Au}}}{\left\langle n S_{1} / S_{n}\right\rangle_{\mathrm{pp}}}\right)^{1 / 4}
$$

Here, $S_{n}$ corresponds to the area occupied by $n$ overlapping strings. Now,

$$
\left\langle\frac{n S_{1}}{S_{n}}\right\rangle=\frac{1}{F^{2}(\xi)},
$$

where $F(\xi)$ is the color suppression factor, which is given as

$$
F(\xi)=\sqrt{\frac{1-e^{-\xi}}{\xi}} .
$$

To calculate the electrical conductivity of strongly interacting matter, which is one of the most important transport properties of QCD matter, we proceed as follows. The mean free path, which describes the relaxation of the system far from equilibrium can be written in terms of the number density and cross section as

$$
\lambda_{\mathrm{mfp}}=\frac{1}{n \sigma_{\mathrm{tr}}},
$$

where $n$ is the number density of an ideal gas of quarks and gluons and $\sigma_{\text {tr }}$ is the transport cross section. In the CSPM, the number density is given by the effective number of sources per unit volume:

$$
n=\frac{N_{\text {sources }}}{S_{n} L} .
$$

Here, $L$ is the longitudinal extension of the string $\sim 1 \mathrm{fm}$. The area occupied by the strings is given by the relation $\left(1-e^{-\xi}\right) S_{n}$. Thus, the effective number of sources is given by the total area occupied by the strings divided by the area of an effective string, $S_{1} F(\xi)$, as shown below:

$$
N_{\text {sources }}=\frac{\left(1-e^{-\xi}\right) S_{n}}{S_{1} F(\xi)},
$$


In general, $N_{\text {sources }}$ is smaller than the number of single strings. $N_{\text {sources }}$ equals the number of strings, $N_{s}$, in the limit of $\xi=0$. So,

$$
n=\frac{\left(1-e^{-\xi}\right)}{S_{1} F(\xi) L}
$$

Now, using Eqs. (5) and (8), we get

$$
\lambda_{\mathrm{mfp}}=\frac{L}{\left(1-e^{-\xi}\right)},
$$

where $\sigma_{\mathrm{tr}}$, the transverse area of the effective strings, equals $S_{1} F(\xi)$.

Now, we derive the formula for electrical conductivity. For this, we use the Anderson-Witting model, in which the Boltzmann transport equation is given as [47]

$$
p^{\mu} \partial_{\mu} f_{k}+q F^{\alpha \beta} p_{\beta} \frac{\partial f_{k}}{\partial p^{\alpha}}=\frac{-p^{\mu} u_{\mu}}{\tau}\left(f_{k}-f_{e q, k}\right),
$$

where $f_{k}=f(x, \vec{p}, t)$ is the full distribution function and $f_{e q, k}$ is the equilibrium distribution function of the $k$ th species. $\tau$ is the mean time between collisions, and $u_{\mu}$ is the fluid 4-velocity in the local rest frame. Equation (10) provides a straightforward calculation of the quark distribution after applying the electric field. The gluon distribution function remains thermal and not altered by electric field. Here, we assume that there are as many quarks (charge $q$ ) as antiquarks (charge $-q$ ) and uncharged gluons in the system. $F^{\alpha \beta}$ is the electromagnetic field strength tensor, which in terms of the electric field and the magnetic flux tensor is given as [48]

$$
F^{\mu \nu}=u^{\nu} E^{\mu}-u^{\mu} E^{\nu}-B^{\mu \nu} .
$$

Since we study the effect of the electric field, the magnetic field is set to zero, $B^{\mu \nu}=0$, in the calculations. The electric current density of the $k$ th species in the $x$ direction is given as

$$
j_{k}^{x}=q_{k} \int \frac{d^{3} p p^{x}}{(2 \pi)^{3} p^{0}} f_{k}=g_{k} \tau \frac{8}{3} \frac{\pi q_{k}^{2} \mathrm{~T}^{2}}{(2 \pi)^{3}} E^{x} .
$$

According to Ohm's law, $j_{k}^{x}=\sigma_{e l} E^{x}$. Using Eq. (12) and relation $n_{k}=g_{k} \mathrm{~T}^{3} / \pi^{2}$, electrical conductivity in the assumption of a very small electric field and no cross-effects between heat and electrical conductivity in the relaxation time approximation is given by

$$
\sigma_{\mathrm{el}}=\frac{1}{3 \mathrm{~T}} \sum_{k=1}^{M} q_{k}^{2} n_{k} \lambda_{\mathrm{mfp}} .
$$

Putting Eq. (9) in Eq. (13) and considering the density of the up quark $(u)$ and its antiquark $(\bar{u})$ in the calculation, we get the expression for $\sigma_{\mathrm{el}}$ :

$$
\sigma_{\mathrm{el}}=\frac{1}{3 \mathrm{~T}} \frac{4}{9} e^{2} n_{q}(\mathrm{~T}) \frac{L}{\left(1-e^{-\xi}\right)} .
$$

Here, the prefactor $4 / 9$ reflects the fractional quark charge squared $\left(\sum_{f} q_{f}^{2}\right)$, and $n_{q}$ denotes the total density of quarks or antiquarks. Here, $e^{2}$ in the natural unit is taken as $4 \pi \alpha$, where $\alpha=1 / 137$.

In the framework of a relativistic kinetic theory, the shear viscosity-to-entropy density ratio, $\eta / s$, is given by [49-51],

$$
\eta / s \simeq \frac{\mathrm{T} \lambda_{m f p}}{5}
$$

In the context of the CSPM, the above equation can be reduced using Eq. (9) as

$$
\eta / s \simeq \frac{\mathrm{T} L}{5\left(1-e^{-\xi}\right)} .
$$

\section{RESULTS AND DISCUSSIONS}

In this section, we discuss the results obtained in the CSPM along with those obtained in various approaches. In Fig. 1, we show $\sigma_{\mathrm{el}} / \mathrm{T}$ as a function of temperature.

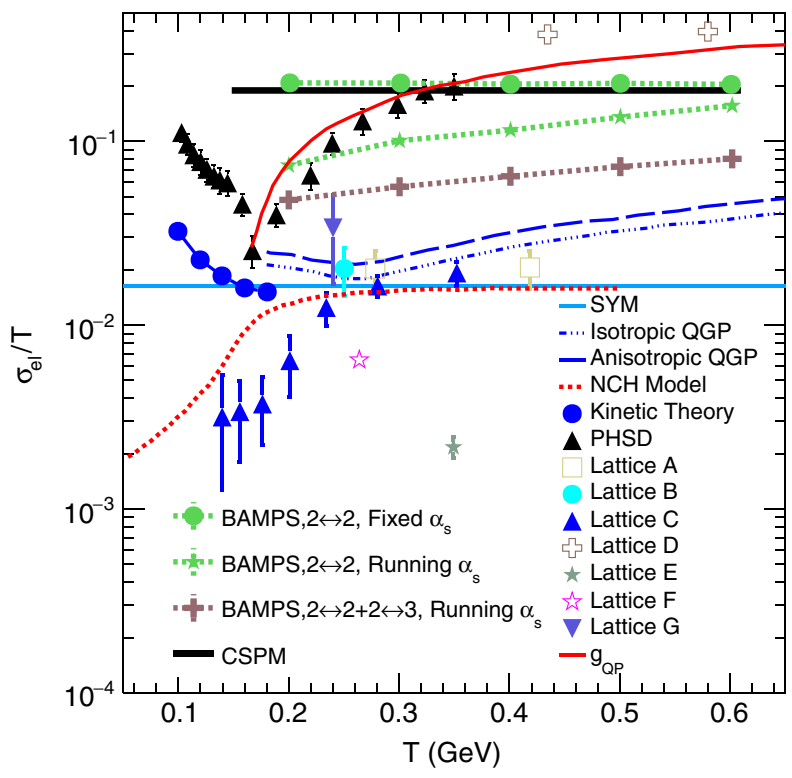

FIG. 1. $\sigma_{\mathrm{el}} / \mathrm{T}$ vs $\mathrm{T}$ plot. The black solid line is the result obtained in the CSPM, and black triangles are PHSD results [16]. The green and brown dotted lines correspond to various BAMPS results [48]. The NCH model [29] results are shown by the red dotted line. The blue circles are kinetic theory calculations [53]. The horizontal line is the result obtained for conformal supersymmetric (SYM) Yang-Mills plasma [54]. Lattice data: lattices A-G [20-26,52] are also shown by various symbols in the figure. The results for isotropic and anisotropic QGP [55] are shown by the blue dash-dotted and dashed lines, respectively. The red solid line depicts the results of the QP model [28]. 
The lattice QCD (IQCD) estimations, i.e., lattices A-G [20-26,52], are shown in the figure for comparison. The green and brown dotted lines are the result of the microscopic transport Boltzmann approach to multiparton scattering (BAMPS) model [48], in which the relativistic $(3+1)$-dimensional Boltzmann equation is solved numerically to extract the electric conductivity for a dilute gas of massless and classical particles described by the relativistic Boltzmann equation. The green dotted line with the solid circles is the result for only elastic processes $2 \leftrightarrow 2$, in which strong coupling constant $\left(\alpha_{s}\right)$ is taken as constant $\left(\alpha_{s}=0.3\right)$ and the green dotted line with the solid stars is with the same setup for running $\alpha_{s}$. The brown dotted line with the brown plus symbols is the BAMPS result, in which both elastic $2 \leftrightarrow 2$ and inelastic $2 \leftrightarrow 3$ processes are taken into consideration with running $\alpha_{s}$. The BAMPS results show a slower increase of $\sigma_{\mathrm{el}} / \mathrm{T}$ with temperature for both the cases of running $\alpha_{s}$ as the effective cross section changes with the temperature, while $\sigma_{\mathrm{el}} / \mathrm{T}$ remains almost independent of temperature for the case of constant $\alpha_{s}$. The BAMPS results are above the $1 Q C D$ results. The solid black line shows our results of the CSPM for $u$ quark and antiquark calculated using Eq. (14). We observe that $\sigma_{\mathrm{el}} / \mathrm{T}$ is almost independent of temperature and matches with the results of BAMPS with constant $\alpha_{s}$, which may be due to the similar basic ingredients and procedure for the estimation of $\sigma_{\mathrm{el}} / \mathrm{T}$.

Although the percolation of the string approach is not directly obtained from QCD, it is QCD inspired, like how the BAMPS model is governed by perturbative QCD. The basic ingredients in the percolation approach are strings, which are stretched between the partons of the projectile and target and forms color electric and magnetic fields in the longitudinal direction. The color strings fragment into $q-\bar{q}$ and/or $q q-\overline{q q}$ pairs and form hadrons [40]. In the present study, we consider the strings to fragment into only $u-\bar{u}$ pairs. We use the Drude formula in the relativistic case to estimate the electrical conductivity, which can be obtained after solving the relativistic Boltzmann transport equation with some approximations as mentioned in Sec. II. So, the observation proclaims the almost similar approach of both the models for the calculation of $\sigma_{\mathrm{el}} / \mathrm{T}$. It has been shown in Ref. [56] that the real electrical conductivity can be even more than 50\% larger than the estimate of the Drude formula unless the cross section is isotropic (no angular dependence).

A nonconformal holographic model ( $\mathrm{NCH})$ [29] is used to estimate the electrical conductivity of the strongly coupled QGP, which is shown by the red dashed line and explains the 1QCD data qualitatively. Kinetic theory [53] is also used to calculate electrical conductivity of hadron gas of which the results are shown by blue circles in the figure, which shows a decrease of $\sigma_{\mathrm{el}} / \mathrm{T}$ with temperature. The electrical conductivity for conformal Yang-Mills plasma [54] is also shown by the horizontal line in the figure. The blue dash-dotted and dotted lines are the results for QGP obtained using the quasiparticle model for the quark and gluons [55] for the isotropic and anisotropic cases, respectively. Here, all the quarks and antiquarks have both the masses, i.e., thermal and bare. The thermal masses of quarks and antiquarks arise due to the interaction with the constituents of the medium. The parton-hadron-string dynamics (PHSD) model results [16] are also shown by the black triangles in the figure for both the phases-hadron gas and quark-gluon plasma with different approaches. The hadron-string-dynamics transport approach has been used for the hadronic sector PHSD, while the partonic dynamics in the PHSD is based on the dynamical quasiparticle model (DQPM). $\sigma_{\mathrm{el}}$ in PHSD decreases with temperature in the hadronic phase when it approaches $\mathrm{T}_{c}$ and increases almost linearly for $\mathrm{T}_{c}<\mathrm{T}$, in the partonic phase after a sudden drop around $\mathrm{T}_{c}$. The calculations of the quasiparticle (QP) model [28] are also shown in the figure by solid red line, which match with the PHSD results for the QGP phase.

Figure 2 shows the variation of $\eta / s$ as a function of $\mathrm{T} / \mathrm{T}_{c}$. Here, $\mathrm{T}_{c}$ is the critical temperature, which is different in different model calculations. The black solid line is the CSPM result, and the broken lines are quasiparticle model results [55]. Here, the dashed line is the result for anisotropic case, while the dash-dotted line is for the isotropic case. A direct comparison with anisotropic QGP gives a feeling of the temperature-dependent effect of anisotropy on the discussed observables in Figs. 2 and 3. However, the comparison with the results for the isotropic case is only

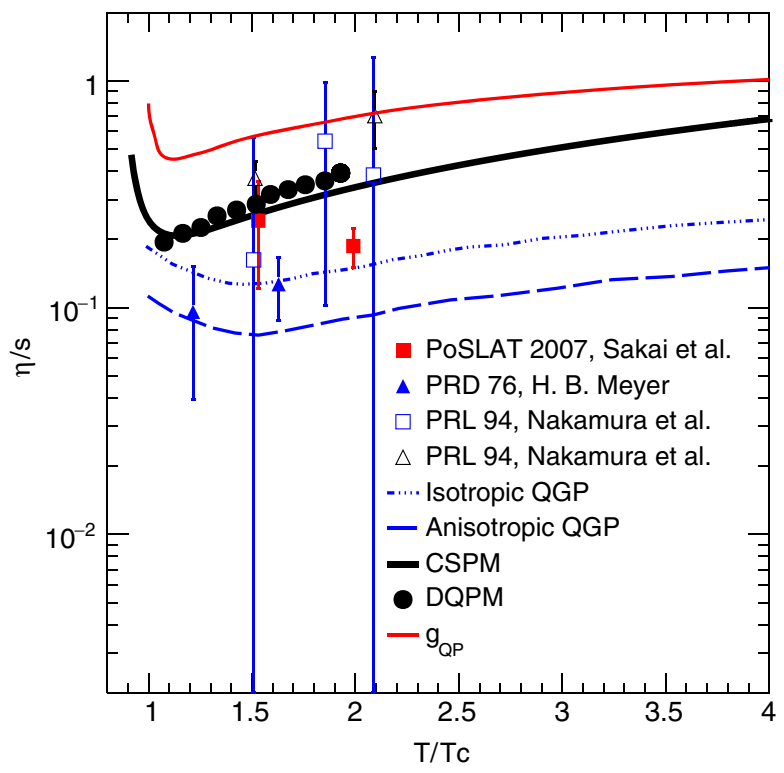

FIG. 2. The ratio $\eta / s$ as a function of $\mathrm{T} / \mathrm{T}_{c}$. The black solid line is the CSPM result, and broken lines are results from Ref. [55]. The symbols are lattice QCD results: full triangles [57], open squares and open triangles [58], and full squares [59]. The black circles are the results obtained in the DQPM [60]. The red solid line is QP model results [28]. 


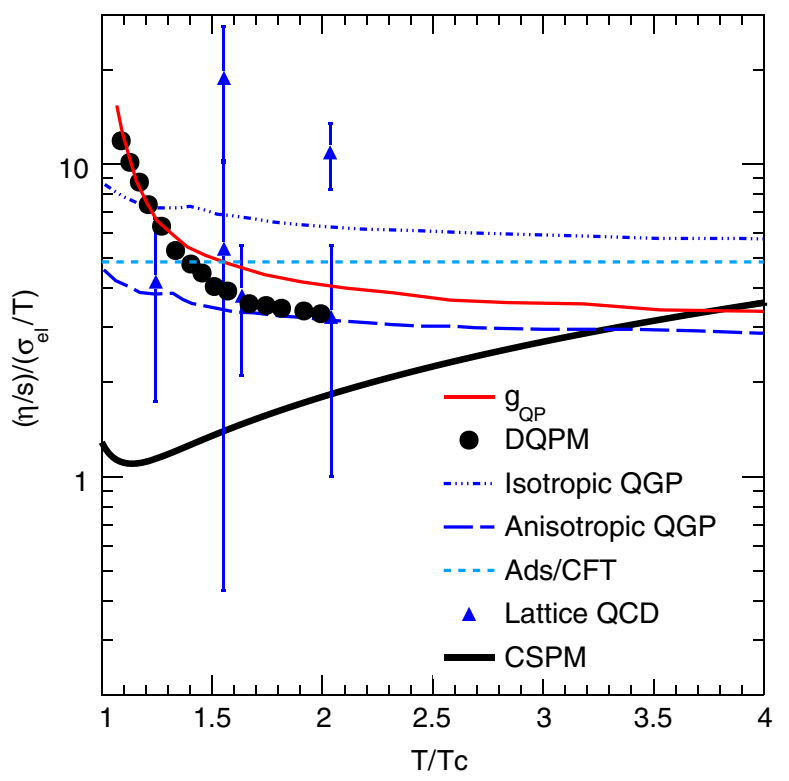

FIG. 3. The ratio $\eta / s$ and $\sigma_{\mathrm{el}} / \mathrm{T}$ with respect to $\mathrm{T} / \mathrm{T}_{c}$. The black solid line is the CSPM result, and broken lines are results from Ref. [55]. The symbols are lattice QCD results [28]. The DQPM and $\mathrm{QP}$ results are shown by the black circles and red solid line, respectively [28].

meaningful for CSPM calculations if the partons are considered massless. The blue triangle symbols are results of $1 Q C D$ with $(2+1)$ dynamical flavors [57-59]. The black circles are the estimations from the DQPM [60]. The red line is the results obtained in the QP model [28]. In the CSPM, $\eta / s$ first decreases, and after reaching a minimum value, it starts increasing with temperature. Thus, it forms a dip that occurs at $\mathrm{T} / \mathrm{T}_{c}=1$. The quasiparticle model results [55] show a similar behavior, but the dip does not occur at the critical temperature in this case. We notice that the CSPM results are close to the DQPM predictions and stay little higher than the results obtained in the quasiparticle model.

Recently, the ratio $(\eta / s) /\left(\sigma_{\mathrm{el}} / \mathrm{T}\right)$ has gained considerable interest in heavy-ion phenomenology [28]. QGP is expected as a good conductor due to the presence of deconfined color charges. A small value of $\eta / s$ suggests large scattering rates that can damp the conductivity. It is observed that $\eta / s$ is affected by the gluon-gluon and quarkquark scatterings while $\sigma_{\mathrm{el}}$ is only affected by the quarkquark scatterings [28]. Thus, the ratio between them is important to quantify the contributions from quarks and gluons in various temperature regions. In this work, we have studied this ratio as a function of temperature using the CSPM. In Fig. 3, we show the ratio of $\eta / s$ and $\sigma_{\mathrm{el}} / \mathrm{T}$ vs
$\mathrm{T} / \mathrm{T}_{c}$. It is observed that this ratio behaves in a fashion similar to $\eta / s$. We have also shown the results obtained for the isotropic and anisotropic QGP using a quasiparticle model [55]. Again, the comparison with the isotropic case is only meaningful. CSPM results are also confronted with the interpolated lattice QCD data [28] and explain the data within error bars. The dotted horizontal line is the AdS/CFT calculation [28] for a strongly coupled system. We also show the results obtained in the DQPM and QP by the black circles and red line, respectively.

\section{SUMMARY AND OUTLOOK}

In summary, we have developed a method to calculate the electric conductivity of strongly interacting matter using the color string percolation approach. We use basically the well-known Drude formula for the estimation of electrical conductivity, which can be obtained after solving the Boltzmann transport equation in relaxation time approximation assuming very small electric fields and no cross-effects between heat and electrical conductivity. We see that the CSPM results for the conductivity stays almost constant with increasing temperature in a fashion similar to that shown by BAMPS data and matches the results obtained in BAMPS with the fixed strong coupling constant considering the elastic cross section only. The CSPM results lie well above the 1QCD results for all the temperatures. We have shown $\eta / s$ as a function of $\mathrm{T} / \mathrm{T}_{c}$ and compared our results with various quasiparticle models for isotropic and anisotropic cases, 1QCD data, DQPM, and QP model results. Similar behavior is found for CSPM results as shown in 1 QCD data and other model predictions. But our results lie above the results obtained from quasiparticle models. CSPM results go in line with that obtained in the DQPM. We have also studied the ratio, $(\eta / s) /\left(\sigma_{\mathrm{el}} / \mathrm{T}\right)$, as a function of $\mathrm{T}$, which behaves in a manner similar to $\eta / s$. We have confronted CSPM results with the results obtained in the quasiparticle model for the isotropic and anisotropic QGP media, 1QCD predictions, estimations from the DQPM, and QP models. The results obtained for electrical conductivity in the CSPM framework validate the outcomes from BAMPS calculations with a fixed strong coupling constant and fail to explain the predictions of $1 \mathrm{QCD}$ data.

\section{ACKNOWLEDGMENTS}

The authors acknowledge the financial support from ALICE Project No. SR/MF/PS-01/2014-IITI(G) of Department of Science and Technology, Government of India. 
[1] M. Gyulassy and L. McLerran, Nucl. Phys. A750, 30 (2005).

[2] C. Gale, S. Jeon, and B. Schenke, Int. J. Mod. Phys. A 28, 1340011 (2013).

[3] B. Schenke, S. Jeon, and C. Gale, J. Phys. G 38, 124169 (2011).

[4] U. Heinz and R. Snellings, Annu. Rev. Nucl. Part. Sci. 63, 123 (2013).

[5] J. Adams et al. (STAR Collaboration), Nucl. Phys. A757, 102 (2005).

[6] R. Kubo, J. Phys. Soc. Jpn. 12, 570 (1957).

[7] S. Plumari, A. Puglisi, F. Scardina, and V. Greco, Phys. Rev. C 86, 054902 (2012).

[8] C. Sasaki and K. Redlich, Phys. Rev. C 79, 055207 (2009).

[9] A. Dobado, F. J. Llanes-Estrada, and J. M. Torres-Rincon, Phys. Rev. D 79, 014002 (2009).

[10] P. Chakraborty and J. I. Kapusta, Phys. Rev. C 83, 014906 (2011).

[11] P. Zhuang, J. Hufner, S. P. Klevansky, and L. Neise, Phys. Rev. D 51, 3728 (1995).

[12] A. Wiranata and M. Prakash, Phys. Rev. C 85, 054908 (2012).

[13] S. Ghosh, Phys. Rev. C 90, 025202 (2014).

[14] Y. Hirono, M. Hongo, and T. Hirano, Phys. Rev. C 90, 021903 (2014).

[15] K. Tuchin, Adv. High Energy Phys. 2013, 490495 (2013).

[16] W. Cassing, O. Linnyk, T. Steinert, and V. Ozvenchuk, Phys. Rev. Lett. 110, 182301 (2013).

[17] K. Fukushima, D. E. Kharzeev, and H. J. Warringa, Phys. Rev. D 78, 074033 (2008).

[18] P. B. Arnold, G. D. Moore, and L. G. Yaffe, J. High Energy Phys. 11 (2000) 001.

[19] P. B. Arnold, G. D. Moore, and L. G. Yaffe, J. High Energy Phys. 05 (2003) 051.

[20] S. Gupta, Phys. Lett. B 597, 57 (2004).

[21] G. Aarts, C. Allton, J. Foley, S. Hands, and S. Kim, Phys. Rev. Lett. 99, 022002 (2007).

[22] P. V. Buividovich, M. N. Chernodub, D. E. Kharzeev, T. Kalaydzhyan, E. V. Luschevskaya, and M. I. Polikarpov, Phys. Rev. Lett. 105, 132001 (2010).

[23] H.-T. Ding, A. Francis, O. Kaczmarek, F. Karsch, E. Laermann, and W. Soeldner, Phys. Rev. D 83, 034504 (2011).

[24] Y. Burnier and M. Laine, Eur. Phys. J. C 72, 1902 (2012).

[25] B. B. Brandt, A. Francis, H. B. Meyer, and H. Wittig, J. High Energy Phys. 03 (2013) 100.

[26] A. Amato, G. Aarts, C. Allton, P. Giudice, S. Hands, and J. I. Skullerud, Phys. Rev. Lett. 111, 172001 (2013).

[27] T. Steinert and W. Cassing, Phys. Rev. C 89, 035203 (2014).

[28] A. Puglisi, S. Plumari, and V. Greco, Phys. Lett. B 751, 326 (2015).

[29] S. I. Finazzo and J. Noronha, Phys. Rev. D 89, 106008 (2014).
[30] S. Mitra and V. Chandra, Phys. Rev. D 94, 034025 (2016).

[31] P. K. Srivastava, L. Thakur, and B. K. Patra, Phys. Rev. C 91, 044903 (2015).

[32] G. D. Moore and J. M. Robert, arXiv:hep-ph/0607172.

[33] G. Baym and H. Heiselberg, Phys. Rev. D 56, 5254 (1997).

[34] D. Fernandez-Fraile and A. Gomez Nicola, Phys. Rev. D 73, 045025 (2006).

[35] N. Armesto, M. A. Braun, E. G. Ferreiro, and C. Pajares, Phys. Rev. Lett. 77, 3736 (1996).

[36] M. Nardi and H. Satz, Phys. Lett. B 442, 14 (1998).

[37] M. A. Braun and C. Pajares, Eur. Phys. J. C 16, 349 (2000).

[38] M. A. Braun, C. Pajares, and J. Ranft, Int. J. Mod. Phys. A 14, 2689 (1999).

[39] M. A. Braun and C. Pajares, Phys. Rev. Lett. 85, 4864 (2000).

[40] M. A. Braun, J. Dias de Deus, A. S. Hirsch, C. Pajares, R. P. Scharenberg, and B. K. Srivastava Phys. Rep. 599, 1 (2015).

[41] J. Dias de Deus and C. Pajares, Phys. Lett. B 642, 455 (2006).

[42] L. J. Gutay, A. S. Hirsch, R. P. Scharenberg, B. K. Srivastava, and C. Pajares, Int. J. Mod. Phys. E 24, 1550101 (2015).

[43] A. S. Hirsch, C. Pajares, R. P. Scharenberg, and B. K. Srivastava, arXiv:1803.02301.

[44] P. Sahoo, S. De, S. K. Tiwari, and R. Sahoo, Eur. Phys. J. A 54, 136 (2018).

[45] P. Sahoo, S. K. Tiwari, S. De, R. Sahoo, R. P. Scharenberg, and B. K. Srivastava, arXiv:1708.06689.

[46] M. A. Braun, J. Dias de Deus, A. S. Hirsch, C. Pajares, R. P. Scharenberg, and B. K. Srivastava, Phys. Rep. 599, 1 (2015).

[47] J. Anderson and H. Witting, Physica (Utrecht) 74, 466 (1974).

[48] M. Greif, I. Bouras, C. Greiner, and Z. Xu, Phys. Rev. D 90, 094014 (2014).

[49] J. Dias de Deus, A. S. Hirsch, C. Pajares, R. P. Scharenberg, and B. K. Srivastava, Eur. Phys. J. C 72, 2123 (2012).

[50] P. Danielewicz and M. Gyulassy, Phys. Rev. D 31, 53 (1985).

[51] T. Hirano and M. Gyulassy, Nucl. Phys. A769, 71 (2006).

[52] G. Aarts, C. Allton, A. Amato, P. Giudice, S. Hands, and J. I. Skullerud, J. High Energy Phys. 02 (2015) 186.

[53] M. Greif, C. Greiner, and G. S. Denicol, Phys. Rev. D 93, 096012 (2016); 96, 059902(E) (2017).

[54] S. Caron-Huot, P. Kovtun, G. D. Moore, A. Starinets, and L. G. Yaffe, J. High Energy Phys. 12 (2006) 015.

[55] L. Thakur, P. K. Srivastava, G. P. Kadam, M. George, and H. Mishra, Phys. Rev. D 95, 096009 (2017).

[56] A. Puglisi, S. Plumari, and V. Greco, Phys. Rev. D 90, 114009 (2014).

[57] H. B. Meyer, Phys. Rev. D 76, 101701 (2007).

[58] A. Nakamura and S. Sakai, Phys. Rev. Lett. 94, 072305 (2005).

[59] S. Sakai and A. Nakamura, Proc. Sci., LATTICE2007 (2007) 221.

[60] R. Marty, E. Bratkovskaya, W. Cassing, J. Aichelin, and H. Berrehrah, Phys. Rev. C 88, 045204 (2013). 